\title{
Optimizing end user QoS in heterogeneous network environments using reputation and prediction
}

\author{
David Giacomini* and Anjali Agarwal
}

\begin{abstract}
Telecommunication consumers are fueling a demand for mobile devices that are rapidly increasing in their capability to provide a wider range of services. These services in turn are consuming more bandwidth and require richer quality of service (QoS) in order to ensure a good end user experience when performing activities such as streaming video content or facilitating voice over IP. As a result, network providers are expanding and improving their coverage area while technology to establish Wi-Fi hotspots is becoming more accessible to every day users. This combination of increase in demand and accessibility, coupled with users' ever-increasing expectations for high quality service presents a growing need to seamlessly optimize the use of the overlaid heterogeneous networks in urban areas to maximize the end user experience via the use of a vertical handover mechanism (VHO). Grey systems theory has been used in a wide range of systems including economic, financial, transportation, and military to accurately forecast time series based on limited information. In this paper, we build on a novel reputation-based VHO decision rating system by proposing the use of the grey model first-order one variable, $G M(1,1)$, in the handover decision making progress. The low complexity of the $\mathrm{GM}(1,1)$ model allows for a quick and efficient prediction of the future reputation score for a given network, providing deeper insight into the current state of the target network. Furthermore, simulations show that the proposed model, in comparison with the original reputation model, improves the decision capability of a mobile node and helps balance the load across the heterogeneous networks employing its strategy.
\end{abstract}

Keywords: Vertical handover (VHO); Heterogeneous networks; Decision; Reputation; Grey model; Grey forecasting; Quality of service (QoS)

\section{Introduction}

Large metropolitan cities are increasingly forming ecosystems for overlaid heterogeneous networks via the co-existence of 3G/4G networks, Wi-Fi hotspots, and WiMAX networks to name a few. Furthermore, mobile devices accessing these networks are exponentially growing in their ability to consume services which are bandwidth and latency intensive, such as video streaming and video chatting. In order to optimize the end user's experience while navigating through this abundance of distinct network access points, the IEEE 802.21 standard [1] was defined, providing a framework for the identification and solicitation of new networks

\footnotetext{
*Correspondence: david.giacomini@gmail.com

Electrical and Computer Engineering Department, Concordia University, Montreal, Quebec H3G 2W1, Canada
}

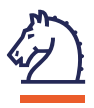

and the mechanism for the actual handover between networks. A key aspect of the vertical handover (VHO) solution which is not covered by this standard is the decision making algorithm itself, which has been the focus of much debate and in which a variety of proposals have been put forth.

In the literature, three general categories of decision making solutions can be observed: solutions based on network conditions, multiple attribute algorithms, and artificial intelligence. In network condition-based solutions, the typical examples leverage the receiver signal strength (RSS) or the signal-to-interference-and-noise ratio (SINR) measured by the mobile node. In [2], the latter was analyzed in relation to the RSS of the mobile node, yielding an overall higher throughput experienced by the mobile node user. The drawback with using the 
network condition approach, as indicated in [3], is that this results in an inefficient power solution as the mobile node must keep both interfaces active in order to measure when the conditions are right to perform the VHO. Furthermore, the quality of service (QoS) extrapolated from the target network by the mobile is not necessarily accurate as there is no understanding of the current load or resource utilization.

In multiple attribute solutions, the authors improve upon the QoS perceived by the mobile node in the network selection process through the use of multiple attribute decision maker (MADM) algorithms which leverage multiple network conditions and performance metrics. In [4], this was accomplished using the analytical hierarchical process (AHP) to assign weights against the alternative networks in relation to the current network, and technique for order preference by similarity to an ideal solution (TOPSIS) was employed to select the best option. In this experiment, criteria such as cost per byte, total bandwidth, allotted bandwidth, utilization, delay, jitter, and packet loss were used to assess the QoS. In comparison with the network condition approach, this method performs much better, but the solution to implement it is much more complex. As a result, this could yield higher delays as the mobile node requires more time to process the algorithms necessary to make the decision, as was observed in [5].

The most popular implementations of VHO decision algorithms using artificial intelligence (AI) are through the use of fuzzy logic or neural networks. In [6], the authors use fuzzy logic in association with a TOPSIS MADM model. In order to make a decision which optimizes the QoS experienced by the user, the TOPSIS model uses resource availability, RSS, mobile node speed, network type, network link business cost, and security as criteria in the analysis. In comparing this technique to the network-condition based solutions, a significant performance gain is seen with the tradeoff resulting in an increased handover delay due to the high complexity of the solution [5].

Through the review of the literature, a novel handover mechanism using reputation scores was observed in the work from Zekri et al. in [7]. In this paper, we build on this scheme by leveraging the grey model first-order one variable $(\operatorname{GM}(1,1))$ prediction algorithm, first proposed in [8] by treating these network scores, which represent the overall QoS of the network, as input into the prediction model. Through using this algorithm, we anticipate that the mobile node will be able to better judge whether the target network is more desirable than the current based on the latest trend in offered QoS and that the heterogeneous ecosystem of networks should be able to better balance the traffic across the networks. In Section 2 , the theory relevant to the reputation model and the
GM $(1,1)$ process is discussed. In Section 3, the details on how the $\operatorname{GM}(1,1)$ algorithm is used in conjunction with the reputation model are presented. Subsequently, Section 4 covers the simulation and network configuration for the experiments, and Section 5 reviews the simulation results. Finally, the conclusion is presented in Section 6.

\section{Related theory}

\subsection{VHO using network reputation}

In order to minimize the computations calculated by the mobile node in the actual decision process, a novel approach was proposed in [7] for a UMTS and WLAN overlaid network. This work serves as the basis of our proposed implementation and as such the theory is reviewed here in detail. The key aspect of this system is that the majority of complex calculations required to facilitate the decision are carried out prior to the actual decision point. This is enabled by defining two types of agents in the overlaid network:

- Mobile reputation agent: Each mobile node in the ecosystem has a reputation agent. The purpose of these agents is to collect real-time performance metrics upon entering and leaving the respective network in order to calculate sample reputation scores. The reputation score is evaluated as either good or bad and then sent to the network reputation agent for aggregation. Furthermore, this agent coordinates in obtaining the current network scores from the target and current network for decision evaluation.

- Network reputation agent: Each distinct network in the ecosystem has one network reputation agent. The purpose of this agent is to aggregate scores received by all the mobile nodes utilizing its associated network and to provide the current aggregate score upon request.

In light of the above, the initial question which comes to mind is 'How does one determine whether the score is good or bad?'. In order to facilitate this choice, the calculated score, $Q_{n}$, must be compared against a threshold score, $Q_{\mathrm{th}}$, which is used as a basis of comparison. The actual and threshold scores themselves are built using the key performance metrics used to quantitatively measure the QoS levels of a network, specifically, bit error rate (ber), delay (del), jitter (jit), and bandwidth (bw). Depending on the class of service $(\mathrm{CoS})$ in question, the $Q_{\text {th }}$ is calculated accordingly, since the minimum requirements of each $\mathrm{CoS}$ vary greatly and put importance on different metrics within the list. As such, since the $Q_{\text {th }}$ is representative of a CoS and does not change, its value can be calculated once beforehand. 
In building the score, a specific weight is assigned to each QoS performance metric, depending on the applicable $\mathrm{CoS}$ in question through the use of the AHP. Specifically, each QoS metric is correlated to a goal of a CoS; subsequently, through the use of the fundamental 1 to 9 AHP scale to denote relative importance [9], the goals are prioritized in relation to each other within a CoS. Assume $p_{i j}$ is the prioritization of Goal $i, G_{i}$, in comparison to Goal $j, G_{j}$, where $\{i, j\} \in\{$ ber, del, jit, bw $\}$, then, as seen in [7]

- $p_{i j}=1$ when the two goals are equal in priority

- $p_{i j}=3$ when $G_{i}$ is weakly more important than $G_{j}$

- $p_{i j}=5$ when $G_{i}$ is strongly more important than $G_{j}$

- $p_{i j}=7$ when $G_{i}$ is very strongly more important than $G_{j}$

- $p_{i j}=9$ when $G_{i}$ is absolutely more important than $G_{j}$.

Based on the above comparisons, the AHP matrix is established and normalized, as can be seen generically across any $\mathrm{CoS}$ in Table 1 . The normalized values, $p_{i j}$, are then used in [8] to calculate the weight, $W_{i}$, associated to a QoS parameter in a given $\mathrm{CoS}$ as

$$
W_{i}=\frac{p_{i 1}+p_{i 2}+p_{i 3}+p_{i 4}}{4} .
$$

Once the weights have been defined, the additional preparatory step required for calculation of the scores is to define normalization factors, $X_{\min }$ or $X_{\max }$, for each of the performance metrics and within each $\operatorname{CoS}$ in order to ensure that the inherent value of the QoS parameter does not drive the score. This can be readily seen when considering that the raw value of jitter is typically in the order of one thousandth, whereas bandwidth can be on the order of one million. The normalizing factor will then be applied to the raw metric, $X_{\text {raw }}$, based on the interpreted worth or cost of the parameter in order to obtain $X_{\text {norm }}$, the normalized value. As discussed in [7], if the parameter is valued more, the lower it becomes, as is the case for jitter, delay, and bit error rate, then the normalizing equation can be seen by (2). Otherwise, if

Table 1 Normalized AHP matrix generalized for a class of service [7]

\begin{tabular}{lcccc}
\hline CoS $_{\mathbf{i}}$ & BER & Delay & Jitter & BW \\
\hline BER & 1 & $p_{12}$ & $p_{13}$ & $p_{14}$ \\
Delay & $1 / p_{12}$ & 1 & $p_{23}$ & $p_{24}$ \\
Jitter & $1 / p_{13}$ & $1 / p_{23}$ & 1 & $p_{34}$ \\
BW & $1 / p_{14}$ & $1 / p_{24}$ & $1 / p_{34}$ & 1 \\
\hline
\end{tabular}

the higher value has more worth, as in the case of bandwidth, the normalizing equation is described by (3)

$$
\begin{aligned}
& X_{\text {norm }}=\frac{X_{\text {min }}}{X_{\text {raw }}} \\
& X_{\text {norm }}=\frac{X_{\text {raw }}}{X_{\text {max }}} .
\end{aligned}
$$

Now, with both the weights and the normalizing factors defined for each QoS performance metric, the $Q_{\text {th }}$ for a given $\operatorname{CoS}, c_{i}$, can then be calculated (as demonstrated in [7]) by normalizing the threshold value of the metric and $\mathrm{CoS}$ in question, and applying the corresponding weight, as seen in (4):

$$
\begin{aligned}
Q_{\mathrm{th}}\left(c_{i}\right)= & W_{\mathrm{ber}\left(c_{i}\right)} \times \frac{\operatorname{ber}_{\text {min }}\left(c_{i}\right)}{\operatorname{ber}_{\mathrm{th}}\left(c_{i}\right)}+W_{\mathrm{del}\left(c_{i}\right)} \\
& \times \frac{\operatorname{del}_{\text {min }}\left(c_{i}\right)}{\operatorname{del}_{\mathrm{th}}\left(c_{i}\right)}+W_{\mathrm{jit}\left(c_{i}\right)} \times \frac{\mathrm{jit}_{\text {min }}\left(c_{i}\right)}{\mathrm{jit}_{\mathrm{th}}\left(c_{i}\right)} \\
& +W_{\mathrm{bw}\left(c_{i}\right)} \times \frac{\mathrm{bw}_{\mathrm{th}}\left(c_{i}\right)}{\mathrm{bw}_{\max }\left(c_{i}\right)} .
\end{aligned}
$$

Similarly, in [7], the actual sample reputation score, $Q_{\mathrm{n}}$ $\left(c_{i}\right)$, is calculated by the mobile node upon entering or leaving a given network in the same fashion as the $Q_{\mathrm{th}}$. The only difference here being that the sample performance metric obtained by the mobile node is normalized instead of the threshold value, as can be seen in (5):

$$
\begin{aligned}
Q_{\mathrm{n}}\left(c_{i}\right)= & W_{\mathrm{ber}\left(c_{i}\right)} \times \frac{\operatorname{ber}_{\text {min }}\left(c_{i}\right)}{\operatorname{ber}_{\mathrm{n}}}+W_{\operatorname{del}\left(c_{i}\right)} \\
& \times \frac{\operatorname{del}_{\text {min }}\left(c_{i}\right)}{\operatorname{del}_{\mathrm{n}}}+W_{\left.\mathrm{jit}_{(}\right)} \times \frac{\mathrm{jit}_{\text {min }}\left(c_{i}\right)}{\mathrm{jit}_{\mathrm{n}}} \\
& +W_{\mathrm{bw}\left(c_{i}\right)} \times \frac{\mathrm{bw}_{\mathrm{n}}}{\mathrm{bw}_{\max }\left(c_{i}\right)} .
\end{aligned}
$$

Once the sample reputation score is calculated, it is compared against the threshold value obtained in (4) and ranked as either good or bad. The ranked score is then sent to the corresponding network-residing agent for aggregation. It is important to note that in [7], the network agent is modeled as processing multiple received scores within a given interval. Furthermore, the network-residing agent can allocate a separate weight for favorable vs. unfavorable scores, $w^{+}$or $w^{-}$, as seen in (6), in order to give more importance to poor network behavior since this is typically of the most concern [7].

$$
r_{\text {sample }}(t)=w^{+} \sum r^{+}(m, n)+w^{-} \sum r^{-}(m, n)
$$

In addition to putting emphasis on the negative scores, the network-residing agent also places greater importance on the recently received scores in [7] via the use of a discounting factor, $\gamma \in[0,1]$. This discount is applied against the summed and weighted new score in (6) and 
then combined with the current network score to form the running aggregate score as seen in (7)

$$
r_{\text {aggr }}(t)=\left\{\begin{array}{ll}
r_{\text {sample }}(t) & , \mid t=1 \\
(1-\gamma) \times r_{\text {aggr }}(t)+\gamma \times r_{\text {sample }}(t), \mid t \geq 2
\end{array} .\right.
$$

The running aggregate score $r_{\mathrm{aggr}}(t)$ is then provided by the network agent upon request from other mobile agents to facilitate handover.

\subsection{GM(1,1) model}

Grey system theory-based models were first introduced by Deng in [8], and since then, they have been used in numerous industries, with their multiple deviations emerging. These variations include the grey Verhulst model, which is beneficial in S-curve response systems [9], and grey residual error correction models, which leverage Fourier series and other feedback mechanisms for systems requiring error correction [10]. The key benefit of the grey system is that only a minimum data set is required to predict an accurate future value. The simplest and most popular implementation of this theory, which is utilized in this paper, is the grey model first-order one variable model, $\operatorname{GM}(1,1)$. This implementation of the grey system must have an input sequence that contains only positive values. In order to model this, let $X^{(0)}$ represent a time series with $n$ values, which is to be analyzed for prediction

$$
X^{(0)}=\left(x^{(0)}(1), x^{(0)}(2), \ldots, x^{(0)}(n)\right), n \geq 4 .
$$

This time sequence is then applied to an accumulation generation operation (AGO) function in order to build $X^{(1)}$ and smoothen the randomness of the original values. This new sequence can be observed in (10) to be constantly growing

$$
\begin{gathered}
X^{(1)}=\left(x^{(1)}(1), x^{(1)}(2), \ldots, x^{(1)}(n)\right), \quad n \geq 4 \\
x^{(1)}(k)=\mathrm{AGO} \times X^{(0)}=\sum_{i=1}^{k} x^{(0)}(i), \\
k=1,2,3, \ldots, n .
\end{gathered}
$$

Subsequently, the AGO-generated sequence is then used to define a mean sequence of adjacent data, $Z^{(1)}$, as follows:

$$
\begin{gathered}
Z^{(1)}=\left(z^{(1)}(1), z^{(1)}(2), \ldots, z^{(1)}(n)\right), \quad n \geq 4, \\
z^{(1)}(k)=\frac{1}{2} x^{(1)}(k)+\frac{1}{2} x^{(1)}(k-1), \\
\quad k=2,3, \ldots, n .
\end{gathered}
$$

From Deng's work in [8], it can be shown that the AGO-generated sequence can be modeled by the first- order differential equation (also known as the whitening equation) in

$$
\frac{d x^{(1)}(t)}{d t}+a x^{(1)}(t)=b
$$

where $a$ and $b$ are referred to as the development coefficient and grey input, respectively. Intuitively, from (10), one can also deduce that

$$
\frac{d x^{(1)}(t)}{d t}=x^{(1)}(k)-x^{(1)}(k-1)=x^{(0)}(k) .
$$

As a result, by substituting (10), (12), and (14) into (13), one can obtain the grey differential equation:

$$
x^{(0)}(k)+a z^{(1)}(k)=b .
$$

In order to solve Equation (15), one must obtain the solution for the $a$ and $b$ parameters. This can be achieved through the use of the least square error method as follows:

$$
[a, b]^{T}=\left(B^{T} B\right)^{-1} B^{T} Y,
$$

where

$$
\begin{aligned}
& \mathrm{Y}=\left[x^{(0)}(2), x^{(0)}(3), \ldots, x^{(0)}(n)\right]^{T}, \\
& B=\left[\begin{array}{cc}
-z^{(1)}(2) & 1 \\
-z^{(1)}(3) & 1 \\
\cdot & \cdot \\
\cdot & \cdot \\
-z^{(1)}(n) & \cdot
\end{array}\right] .
\end{aligned}
$$

Once $[\mathrm{a}, \mathrm{b}]$ is solved and knowing that the initial condition is $x^{(1)}(0)=x^{(0)}(1)$, the solution to the first-order differential equation is

$$
x_{\mathrm{p}}^{(1)}(k+1)=\left[x^{(0)}(1)-\frac{b}{a}\right] e^{-a k}+\frac{b}{a},
$$

where $x_{\mathrm{p}}^{(1)}$ is the AGO-generated value at the predicted time $k+1$. As such, in order to determine the predicted value at $k+1$, the inverse AGO is applied against (19) in order to obtain

$$
x_{\mathrm{p}}^{(0)}(k+1)=\left[x^{(0)}(1)-\frac{b}{a}\right] e^{-a k}\left(1-e^{a}\right) .
$$

Additionally, the solution defined by (20) can be expanded to obtain a predicted value at time $(k+H)$ :

$$
x_{\mathrm{p}}^{(0)}(k+H)=\left[x^{(0)}(1)-\frac{b}{a}\right] e^{-a(k+H-1)}\left(1-e^{a}\right) .
$$




\section{Proposed model: VHO using network reputation and $\mathrm{GM}(1,1)$ predictions}

Numerous approaches have been reviewed in the pursuit of facilitating a VHO decision module that results in not only minimal delay during the handover process but also making a choice that provides the most benefit to the applications currently in use by the mobile device. As a result, in order to improve upon the novel approach presented in [7] without impacting the efficiency of the decision making duration, the network calculated aggregate scores are used as an input into the $\operatorname{GM}(1,1)$ algorithm to predict the trend in which the network performance is heading. The purpose of this enhancement is to provide advance knowledge of the state of the network to the mobile node so that they can make a more informed decision.

In building the proposed model, several other modifications were made to the original model proposed in [7]. First, in reviewing (5) and (6), it can also be noted that the $Q_{\mathrm{n}}\left(c_{i}\right)$ calculated value provides a much richer representation of the overall network experience than a binary transformed rate. As such, we propose to use the calculated $Q_{\mathrm{n}}\left(c_{i}\right)$ as the input into the aggregation function. Assuming that the network-residing agent will then calculate the aggregate score sequentially upon receipt of a score from the mobile agent (in a first-in-first-out manner), Equation (6) can be refactored as

$$
r_{\text {sample }}(t)=\left\{\begin{array}{c}
w^{-} Q_{\mathrm{n}}\left(c_{i}\right), Q_{\mathrm{n}}\left(c_{i}\right)<Q_{\mathrm{th}}\left(c_{i}\right) \\
w^{+} Q_{\mathrm{n}}\left(c_{i}\right), Q_{\mathrm{n}}\left(c_{i}\right) \geq Q_{\mathrm{th}}\left(c_{i}\right)
\end{array} .\right.
$$

Additionally, in order to ensure that the calculated aggregate reputation score represents the most current state of the network as closely as possible, we propose to instruct the mobile agents to poll for the $Q_{\mathrm{n}}\left(c_{i}\right)$ the moment the mobile agent enters the network and then periodically until it leaves.

Upon receiving the sample score, the network-residing agent will then immediately calculate the running aggregate network score instead of computing the difference of mobile nodes experiencing good service vs. those experiencing bad service. The reasoning behind this is that, to be consistent, it is preferable that each reported sample score communicated by a mobile node affects the overall computed network reputation score in the same fashion. By first combining the scores in a given period, one mobile user's sample score in one interval could potentially have a different impact on the computed network score if the same score was leveraged in another interval. In this way, instead of combining the received scores within an interval, the network-residing reputation agent in the proposed model queues sample scores and processes them sequentially in a first-in-first-out (FIFO) manner.

As a result, when a mobile node approaches a potential target network, instead of requesting the current aggregated reputation score, we propose that the mobile node requests the predicted future aggregate score. If the predicted score of the target network is greater than the predicted score of the current network and the acceptable threshold, a handover is initiated. Otherwise, the mobile node remains in the current network, as presented in Figure 1. This handover process is considered the passive process in our simulation, as the handover investigation is triggered by the $802.21 \mathrm{MIH}$ link detection events (i.e., not actively by the mobile node itself). If the mobile node's RSS within the current network drops below a given threshold, which can be configured within the MIH protocol, a link going down event is received, forcing the mobile node to search for an available network to handover to before the connection is lost. This is considered the forced handover process.

\section{Simulation and network configuration}

The simulation design and execution was carried out using the open-source discrete-event network simulation tool NS2. Modeling of the WiMAX entities and 802.21 protocol was made possible via the Mobility module, developed in the 'Seamless and Secure Mobility' project by the National Institute of Science and Technology (NIST, Gaithersburg, USA) [11], and the modeling of the UMTS entities was made possible via the EURANE module, developed by the European Commission (Brussels, Belgium) 5th Framework Project, 'SEACORN' [12]. An MPEG traffic generation module was leveraged from [13] in order to simulate streaming video or voice.

In order to measure the performance of the proposed model vs. the original model defined in [7], several experiments were carried out. The first category of simulations pertained to observing one mobile node against varying network conditions using the two models, while the second category of simulations involved observing the overall network ecosystem while a multitude of mobile nodes interacted in the network under varying network conditions using the two models. In both categories, the layouts included UMTS ubiquitous coverage with a WiMAX network (range $500 \mathrm{~m}$ ) overlapped with a Wi-Fi network (range $100 \mathrm{~m}$ ). Additionally, the default network scores assigned to these networks were set to prioritize the networks in the following order: Wi-Fi, WiMAX, and UMTS, with the latter being given the lowest priority due to its typically higher cost.

Furthermore, in the second category of experiments, the mobile nodes were configured to travel at three different 


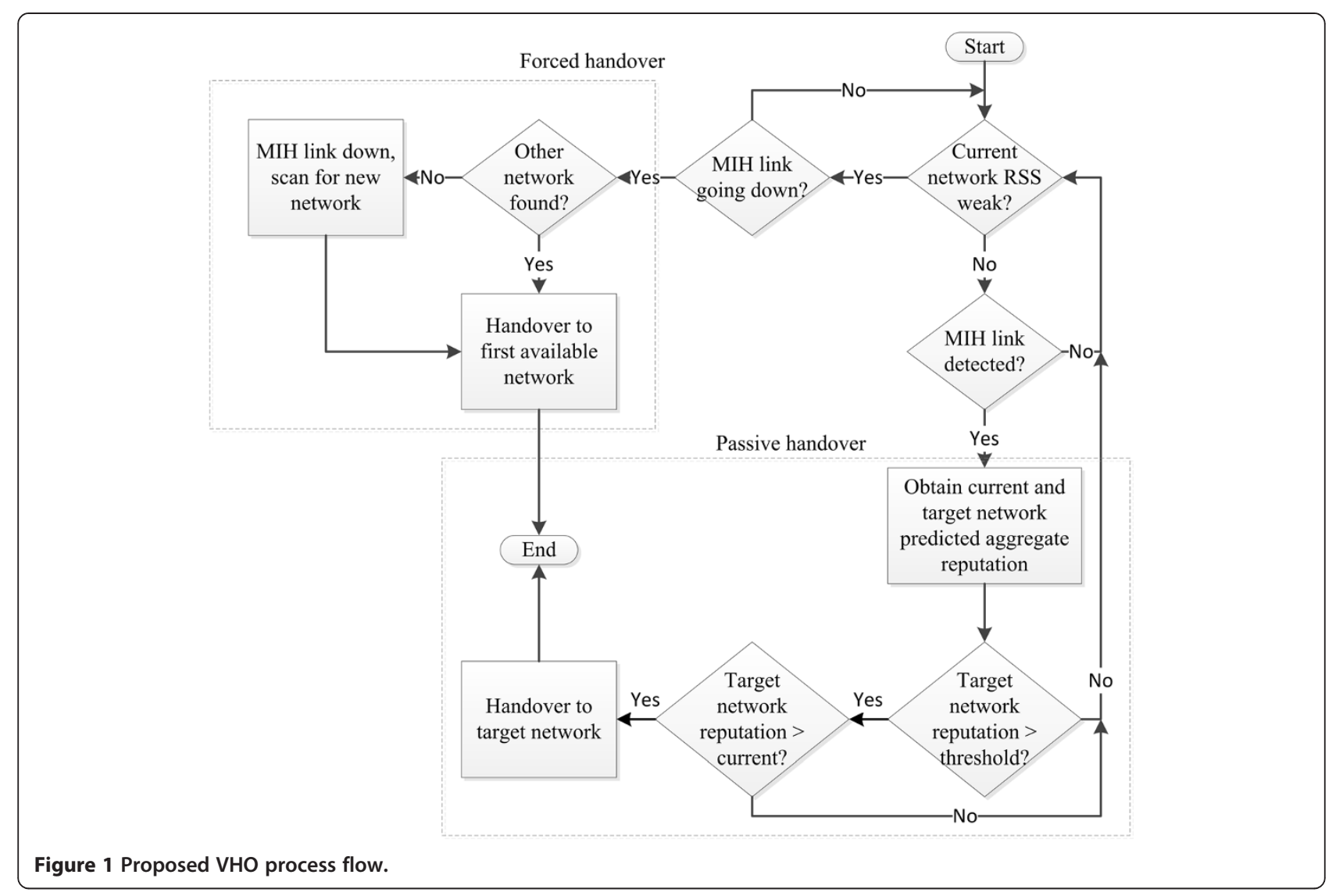

types of speeds in order to simulate the different methods of urban travel:

- Walking - $5 \mathrm{~km} / \mathrm{h}, 1.38 \mathrm{~m} / \mathrm{s}$

- Riding the bus - $40 \mathrm{~km} / \mathrm{h}, 11.11 \mathrm{~m} / \mathrm{s}$

- Riding in the car - $75 \mathrm{~km} / \mathrm{h}, 19.4 \mathrm{~m} / \mathrm{s}$.

Finally, the threshold values were defined using the minimum values required for conversational CoS transmission, and the weights were built using the values calculated in [14], which can be seen in Table 2.

\section{Simulation results}

\subsection{Single mobile node}

\subsubsection{Scenario 1: gradually varying traffic}

The first experiment involving a single node considers the gradual increase and decrease of traffic as a node

Table 2 Weights calculated in [12] for each performance metric, per CoS

\begin{tabular}{lcccc}
\hline Traffic class & BER & Delay & Jitter & Bandwidth \\
\hline Conversational & 0.04998 & 0.45002 & 0.45002 & 0.04998 \\
Streaming & 0.03737 & 0.11380 & 0.42441 & 0.42441 \\
Interactive & 0.63593 & 0.16051 & 0.04304 & 0.16051 \\
Background & 0.66932 & 0.05546 & 00546 & 0.21976 \\
\hline
\end{tabular}

traverses from one network into another, as can be seen in the network topology depicted in Figure 2. In this scenario, the traffic applied to the Wi-Fi network is controlled in order to produce gradually varying scores. The mobile node is iteratively made to traverse from the WiMAX to the Wi-Fi zone at different times throughout the varying load curve, as seen in Figure 3. As a result of the gradual variance, the mean error between the predicted score for time $t$ (calculated at $t-1 \mathrm{~s}$ ), and the actual aggregate score observed at time $t$ is $9.85 \%$.

From the figure, it can be seen that as the network becomes congested, the mobile node using the predicted score at time $(t)$ has advanced warning that the QoS of the network is deteriorating. This can be seen at time $t=35 \mathrm{~s}$ and $t=40 \mathrm{~s}$, where the aggregate scores are above the threshold and perceived as good, while the predicted scores are below the threshold and perceived as bad. Since the aggregate scores following the above time instances do fall beneath the threshold, the predicted scores allow the mobile node to make the VHO decision sooner and maintain an increased overall experience.

\subsubsection{Scenario 2: traffic spike}

The second experiment carried out against a single node considers a spike occurring within the traffic flow. In this scenario, the traffic applied to the Wi-Fi network is 


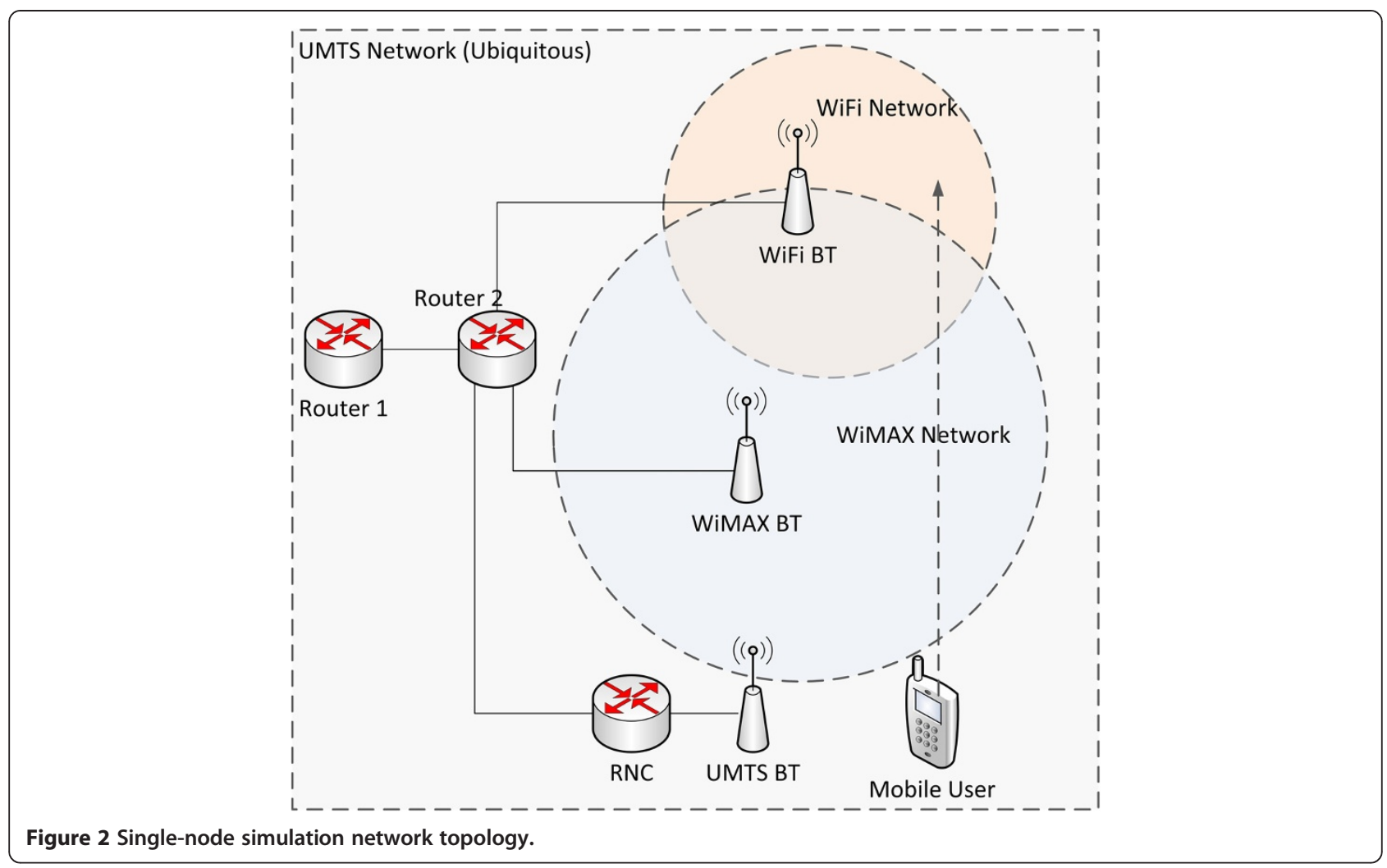

controlled to produce gradually varying scores with a sudden surge introduced temporarily and then removed. Similarly to the first experiment, the mobile node is iteratively made to traverse from the WiMAX to the Wi-Fi zone at different times throughout the varying load curve, as seen in Figure 4.

As is evident from the figure, the spike in traffic corresponds to the sudden drop in the aggregate score at $t=35 \mathrm{~s}$. Furthermore, it is clear that the predicted score does not provide any additional benefits in the case of a sudden degradation of the network and in fact yields a very large mean error of $38.78 \%$. This makes sense as there are no prior values that can give advanced warning of the sudden change in traffic load. The advantage can be seen when the QoS is re-established at time $t=55 \mathrm{~s}$. Instead of deciding to join on the first

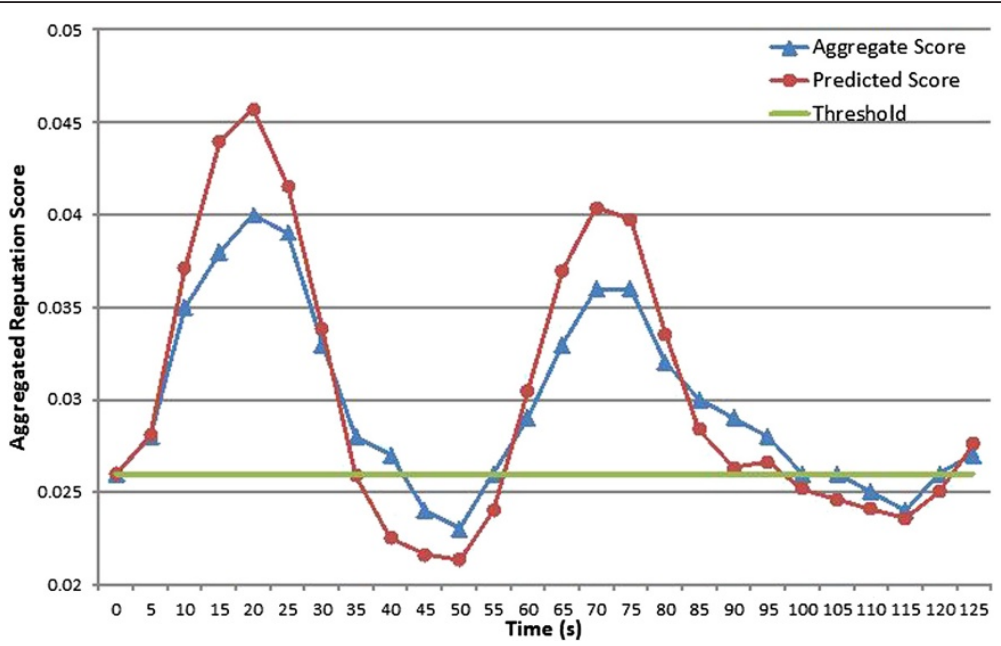

Figure 3 Comparison of sampled reputation scores with gradually varying traffic load. Comparison of sampled reputation scores over time between proposed prediction model and existing aggregate model. 


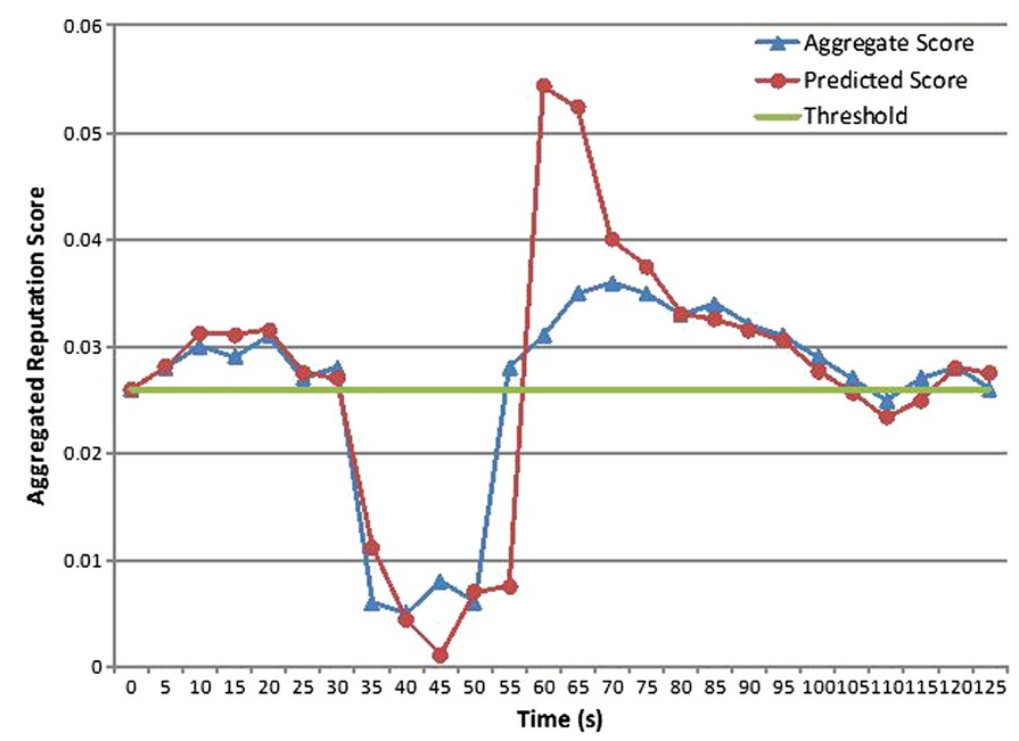

Figure 4 Comparison of sampled reputation scores with traffic load spike. Comparison of sampled reputation scores over time between proposed prediction model and existing aggregate model.

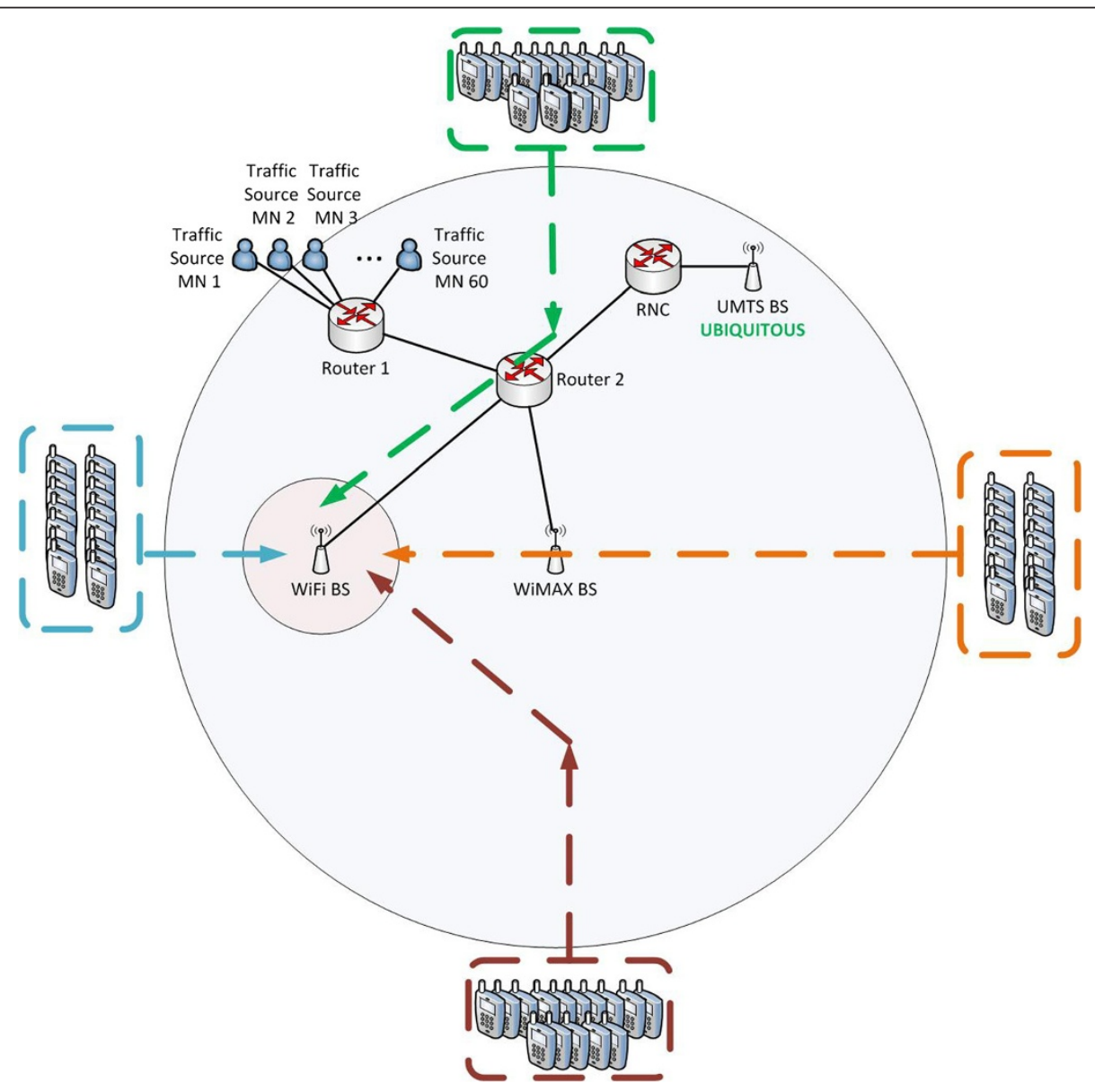

Figure 5 Topology and mobile node path for WiMAX and Wi-Fi traffic overload scenario. 

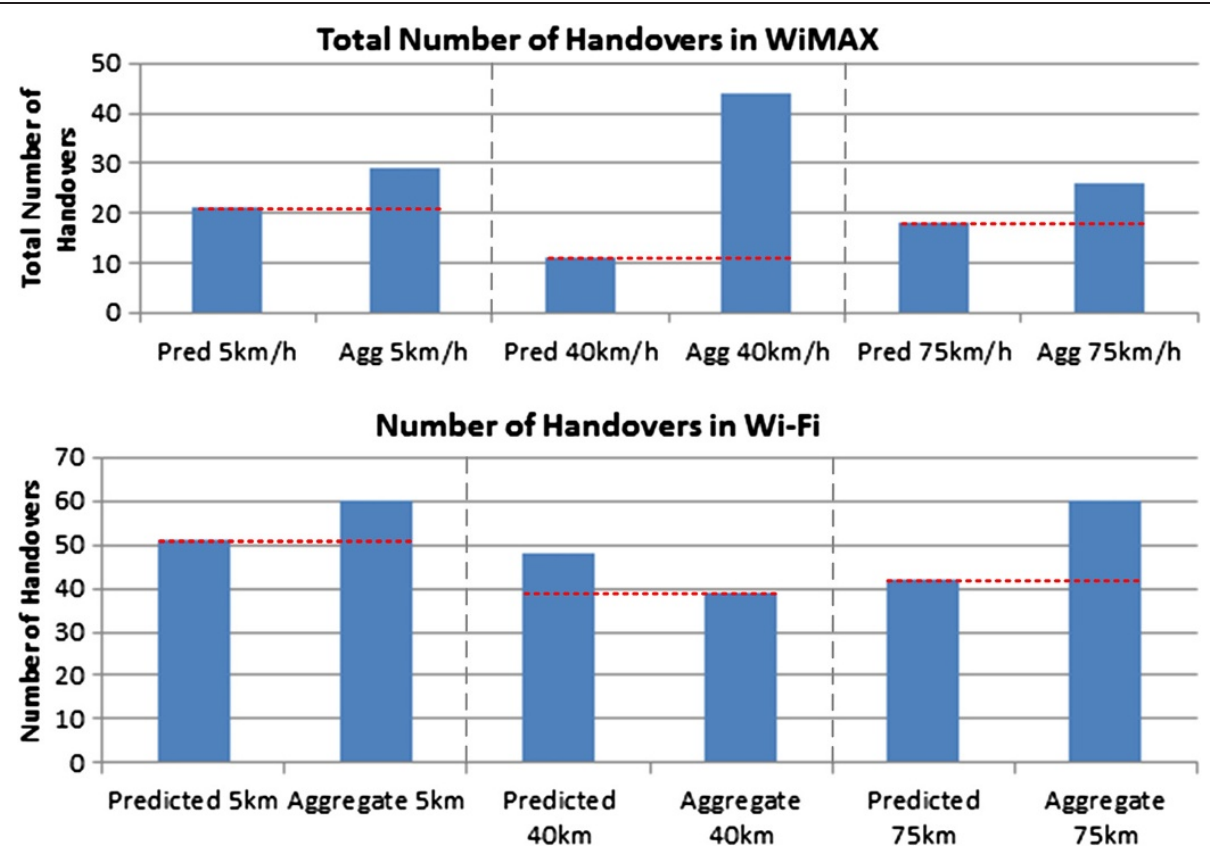

Figure 6 Comparison of total number of handovers between the proposed predicted and original aggregate models. Comparison of total number of handovers in WiMAX (top) and Wi-Fi (bottom) between the proposed predicted and original aggregate models throughout the simulation in scenario 1 for multiple nodes, across all speeds.

instance of a perceived good score, as in the case of the aggregate score, the predicted score requires the occurrence of at least two 'good' scores. This enables the mobile node to make a more confident VHO decision and help reduce the number of unnecessary handovers overall in the network.

\subsection{Multiple mobile nodes}

\subsubsection{Scenario 1: traffic overload}

The first scenario leveraging multiple nodes consisted of 60 mobile nodes rapidly overloading the WiMAX network followed by overloading the Wi-Fi network, as seen in Figure 5. The summary of the total number of handovers into the WiMAX and Wi-Fi network over the course of the entire simulation at the three different speeds is shown in Figure 6. As can be observed, in this scenario, the proposed prediction model results in fewer handovers to either network type at all speeds, except for handovers made to the Wi-Fi network at $40 \mathrm{~km} / \mathrm{h}$. Apart from the exception scenario just mentioned, this aligns to the expected results due to the fact that since the networks are being quickly overloaded, the prediction model is providing advanced notice to the mobile node, allowing more nodes to opt out of the handover.

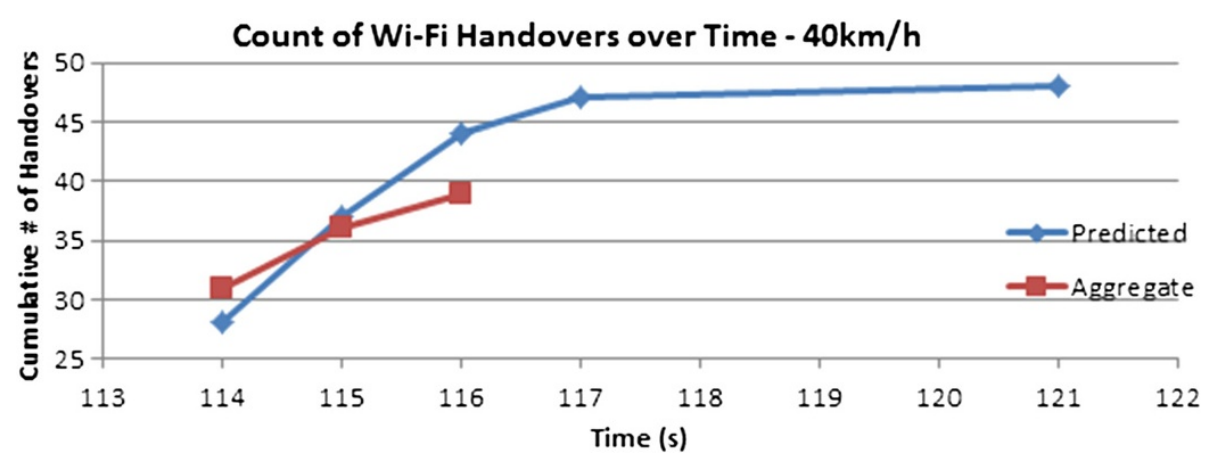

Figure 7 Comparison of the cumulative number of handovers over time. Comparison between the proposed prediction model and the existing aggregate model at $40 \mathrm{~km} / \mathrm{h}$ in the Wi-Fi network. 


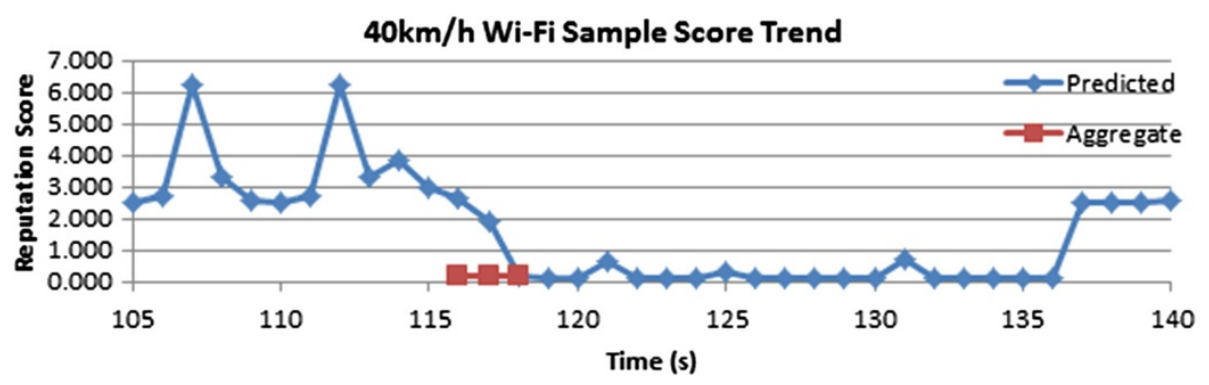

Figure 8 Comparison of the reputation scores while within the Wi-Fi network with traffic overload. The scores are sampled by the mobile nodes while within the Wi-Fi network at $40 \mathrm{~km} / \mathrm{h}$, between the prediction model and aggregate model.

Upon investigating the exception scenario further, it can be seen that the reason why there are more handovers at $40 \mathrm{~km} / \mathrm{h}$ into Wi-Fi under the proposed prediction model is the fact that there were very few handovers made originally to WiMAX. As a result, at $40 \mathrm{~km} / \mathrm{h}$, the majority of the nodes remained in UMTS when reaching WiMAX, and since UMTS had the lowest network score between the three networks, when these nodes arrived at the Wi-Fi hotspot, they more readily perceived the Wi-Fi network as better and thus joined. This is supported by Figures 7, 8, and 9 as follows. In Figure 7, we see that the number of handovers to $\mathrm{Wi}-\mathrm{Fi}$ in the prediction model exceeds the original model at around $115 \mathrm{~s}$; subsequently, in Figure 8, the sample scores in the prediction model are higher at around $115 \mathrm{~s}$, while the WiMAX values are substantially lower in Figure 9. As a result, given that the UMTS score is slightly above the threshold, around 0.075, and the Wi-Fi scores are initially around 3 , the majority of nodes handover.

\subsubsection{Scenario 2: increase and decrease of traffic}

In the second scenario, the WiMAX and Wi-Fi networks are more gradually overloaded; towards the end of the simulation, there is a reduction in traffic in the $\mathrm{Wi}-\mathrm{Fi}$ network as mobile nodes are still joining, as shown in
Figure 10. Given this, the expectation is that again, in WiMAX there would be fewer handovers, while in Wi-Fi, there would be more due to the decrease in traffic.

After simulating the scenario at the three different speeds, the results can be seen in Figure 11. Contrary to the expected results, there were more handovers permitted via the proposed prediction model in WiMAX and fewer handovers in Wi-Fi. In reviewing the $40-\mathrm{km} / \mathrm{h}$ instance, for WiMAX, it is observed that due to the gradual increase in traffic, the prediction curve is not as steep, permitting more handovers in the prediction model, as seen by the sample scores calculated by the mobile nodes in Figure 12. In Wi-Fi, it is observed that the prediction model requires numerous positive results in order to result in an advertised score with increased value; this is due to the fact that while some of the nodes begin experiencing better QoS when the traffic slowdown occurs, there are still numerous nodes experiencing poor service, and the average of all the calculated scores still tends to be low, as see in Figure 13.

\subsubsection{Prediction error}

When comparing the predicted score against the actual score in scenario 1 , there is a mean error of $76.23 \%$

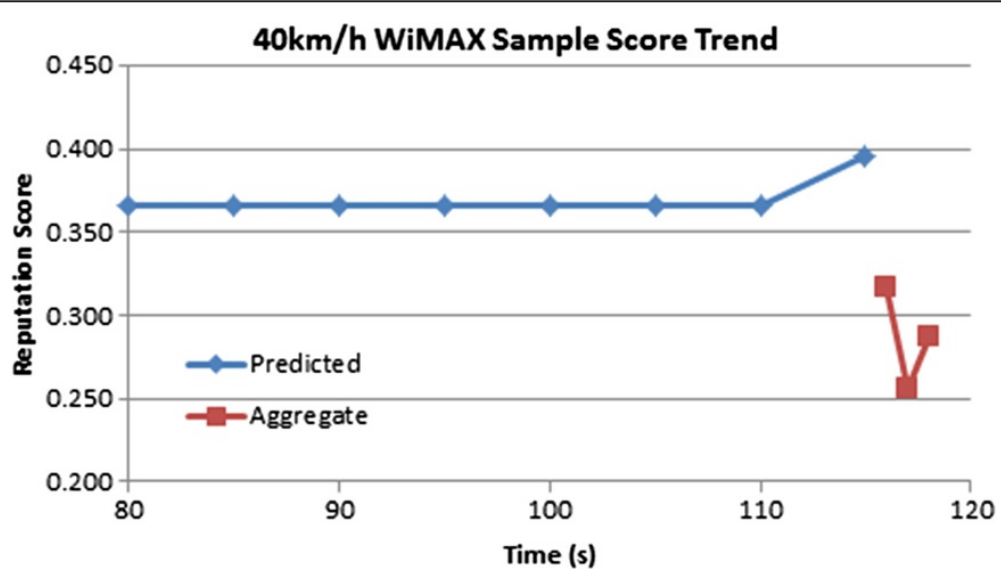

Figure 9 Comparison of the reputation scores while within the WiMAX network with traffic overload. The scores are sampled by the mobile nodes while within the WiMAX network at $40 \mathrm{~km} / \mathrm{h}$, between the two models. 


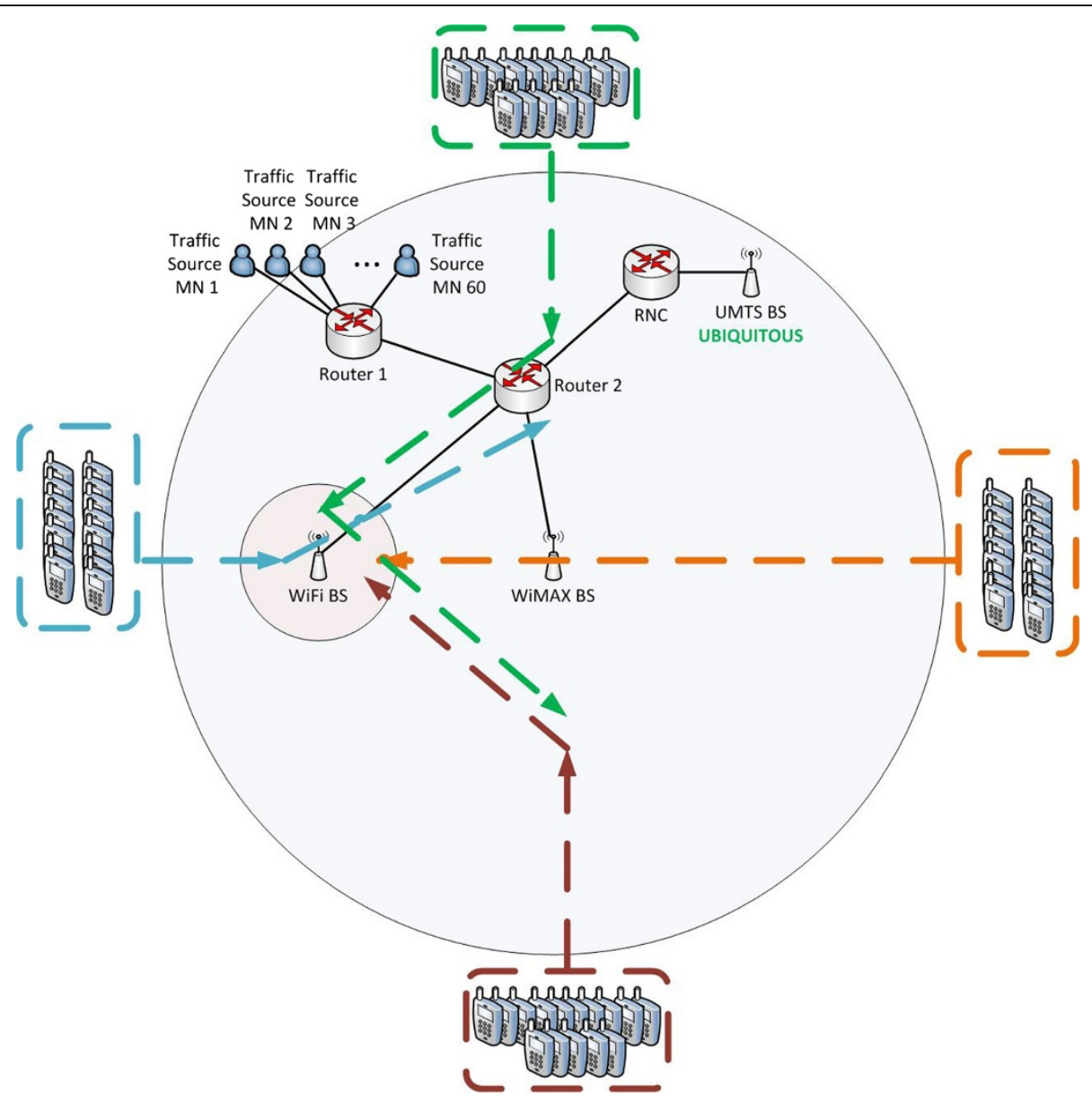

Figure 10 Topology and mobile node path for WiMAX and Wi-Fi with increasing and decreasing traffic scenarios.
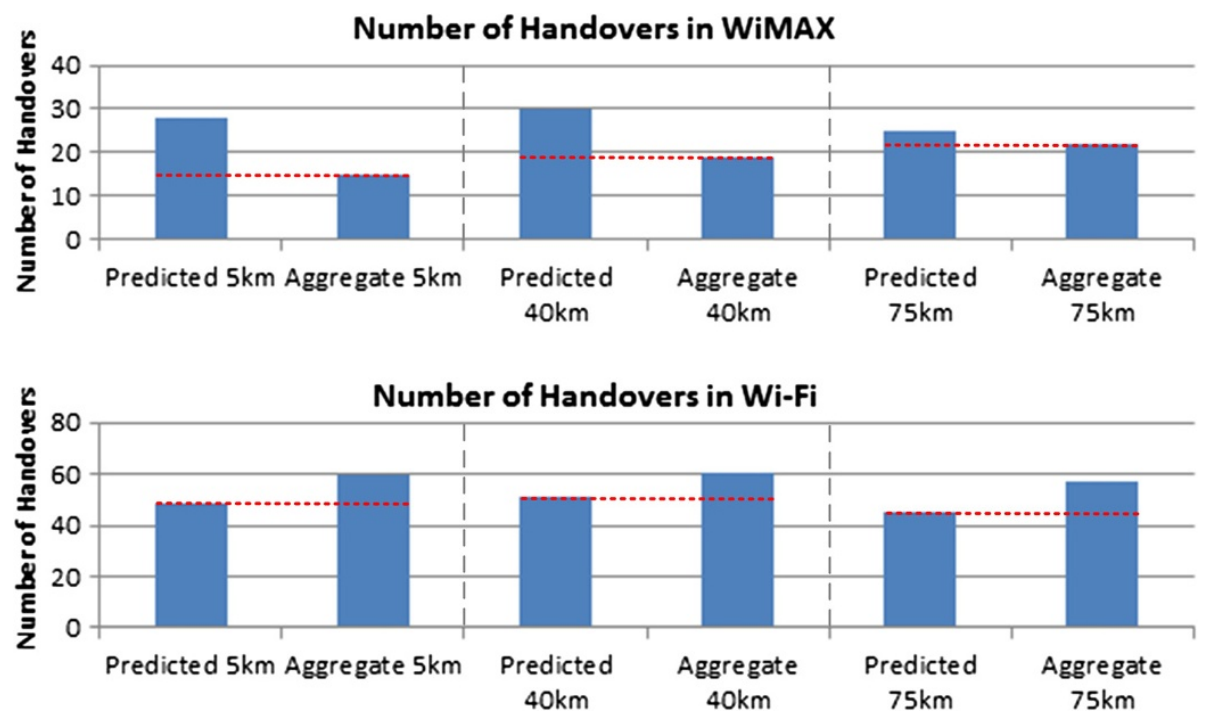

Figure 11 Comparison of the total number of handovers in WiMAX (top) and Wi-Fi (bottom). The proposed predicted and original aggregate models are compared throughout the simulation in scenario 2 for multiple nodes, across all speeds. 


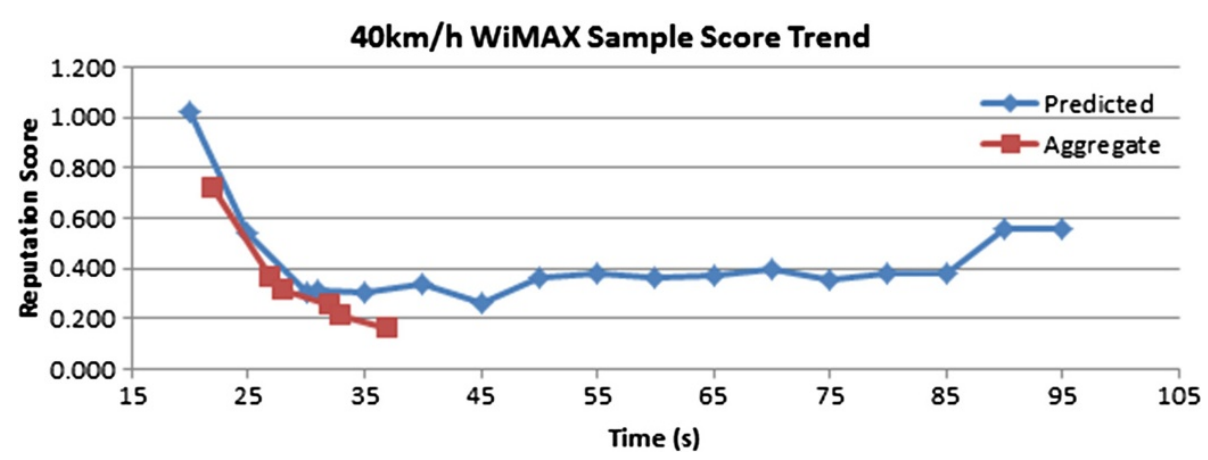

Figure 12 Comparison of the reputation scores while within the WIMAX network with varying traffic. The scores are sampled by the mobile nodes while within the WiMAX network at $40 \mathrm{~km} / \mathrm{h}$, between the prediction model and aggregate models.

across the three different speeds. Similarly, in scenario 2, the prediction error sits at $62.06 \%$ across all speeds. In both scenarios, this can be attributed to the fact that the prediction model is generating significantly more samples, which impact the prediction algorithm described in the $\operatorname{GM}(1,1)$ model. This can be seen in Figure 8, where only three samples are obtained for the original model in a given timeframe while the prediction model has roughly ten times the sample data, and in Figure 13, where the significantly higher sample counts keep the prediction curve much lower than the aggregate.

\section{Conclusion}

In synopsis, in this paper, we have investigated the performance of an improved $\mathrm{VHO}$ reputation model based on a reputation system proposed in [7] and extended by the $\operatorname{GM}(1,1)$ algorithm. In comparing the proposed algorithm vs. the original model with respect to a single node and a network ecosystem, clear improvements can be seen in both situations. From a single-mobile-node perspective, there is an advanced warning of network degradation and an increased hold-off time during surge in network load, preventing needless handovers. From a network perspective, the proposed model reduces the number of handovers between heterogeneous networks under rapid congestion scenarios, allowing the traffic to be well balanced in the ecosystem. In gradually varying network conditions, the prediction model will have a much diminished impact on the overall number of handovers so long as the target network is providing a sufficient quality of service. Furthermore, as in the single-mobile-node case, it reduces the number of handovers during sudden reductions in traffic, diminishing the possibility of a handovers when the network has not yet stabilized. Our future work includes exploring the advantages of using this enhanced model in comparison with MADM and artificial intelligence solutions, investigating the benefit of using other grey model systems such as the grey residual error correction model, and reducing the amount of score noise observed in the results.

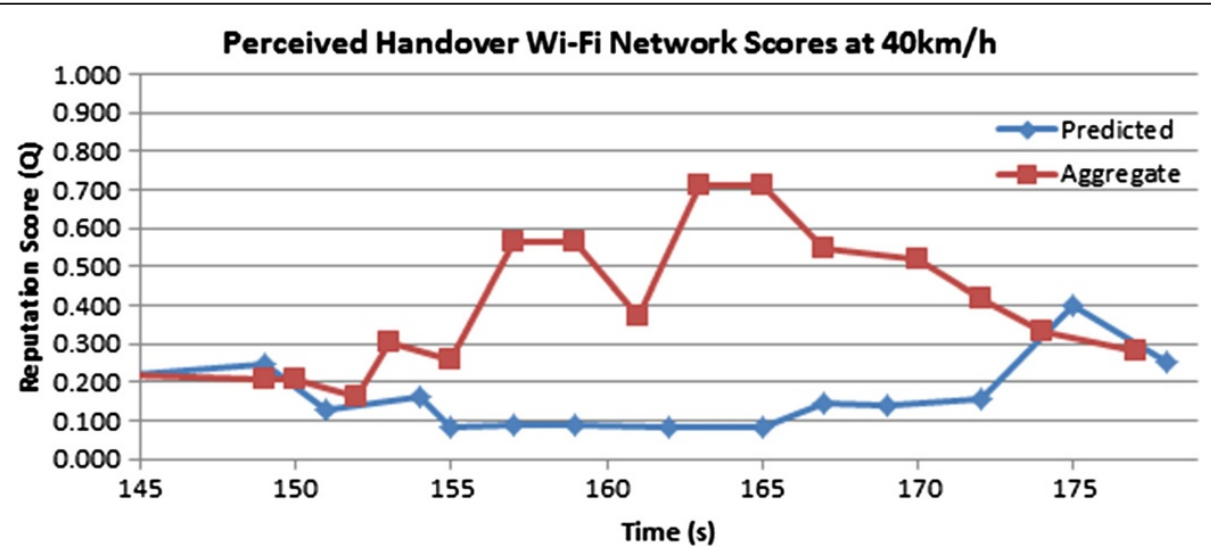

Figure 13 Comparison of the reputation scores while within the Wi-Fi network with varying traffic. The scores are sampled by the mobile nodes upon entering the Wi-Fi network at $40 \mathrm{~km} / \mathrm{h}$, between the prediction model and aggregate models. 


\section{Competing interests}

Both authors declare that they have no competing interests.

Received: 31 October 2012 Accepted: 23 October 2013

Published: 1 November 2013

\section{References}

1. IEEE, Standard for Local and Metropolitan Area Networks_Part 21: Media Independent Handover. IEEE Std 802.21-2008 (IEEE, New York, 2009)

2. AA Bathich, MD Baba, M Ibrahim, IEEE 802.21 based vertical handover in WiFi and WiMAX networks. Paper presented at the IEEE symposium on computers and informatics (ISCI), Penang, Malaysia, 18-20 Mar 2012

3. A Nyamapfene, B Naeem, Seamless vertical handover in Wi-Fi and WiMAX networks using RSS and motion detection: an investigation. Pac. J. Sci. Technol. 12(1), 298-304 (2011)

4. L Mohamed, C Leghris, A Adib, A hybrid approach for network selection in heterogeneous multi-access environments. Paper presented at the 2011 4th IFIP international conference on new technologies, mobility and security (NTMS), Paris, France, 7-10 Feb 2011

5. AA Yussuf, WH Hassan, S Issa, A review of VHD approaches in next generation wireless networks. Paper presented at the second international conference on digital information and communication technology and its applications (DICTAP), Bangkok, Thailand, 16-18 May 2012

6. MM Alkhawlani, KA Alsalem, AA Hussein, Multi-criteria vertical handover by TOPSIS and fuzzy logic. Paper presented at the international conference on communications and information technology (ICCIT), Aqaba, Jordan, 29-31 Mar 2011

7. M Zekri, B Jouaber, D Zeghlache, On the use of network QoS reputation for vertical handover decision making. Paper presented at the IEEE 2010 GLOBECOM workshops on handover and network selection in future broadband networks, Miami, Florida, USA, 6-10 Dec 2010

8. JL Deng, Introduction to grey system theory. J. Grey System 1(1), 1-24 (1989)

9. W Zhengxin, D Yaoguo, W Yemei, A new grey Verhulst model and its application. Paper presented at the IEEE international conference on grey systems and intelligent services, Nanjing, China, 18-20 Nov 2007

10. E Kayacan, B Ulutas, O Kaynak, Grey system theory-based models in time series prediction. Expert Syst. Appl. 37(2), 1784-1789 (2010)

11. NIST, Seamless and secure mobility homepage, http://www.nist.gov/itl/ antd/emntg/ssm_tools.cfm. Accessed 1 Sept 2010

12. EURANE, The EURANE homepage, http://eurane.ti-wmc.n//. Accessed 1 Sept 2010

13. A Matrawy, I Lambadaris, MPEG4 traffic source homepage, http://www.sce. carleton.ca/ amatrawy/mpeg4/. Accessed 1 Sept 2010

14. E Stevens-Navarro, VWS Wong, Comparison between Vertical Handoff Decision Algorithms for Heterogeneous Wireless Networks. Paper presented at the IEEE 63rd vehicular technology conference (VTC), Melbourne, Australia, 7-10 May 2006

doi:10.1186/1687-1499-2013-256

Cite this article as: Giacomini and Agarwal: Optimizing end user QoS in heterogeneous network environments using reputation and prediction. EURASIP Journal on Wireless Communications and Networking 2013 2013:256.

\section{Submit your manuscript to a SpringerOpen ${ }^{\circ}$ journal and benefit from:}

- Convenient online submission

- Rigorous peer review

- Immediate publication on acceptance

- Open access: articles freely available online

- High visibility within the field

- Retaining the copyright to your article

Submit your next manuscript at $\gg$ springeropen.com 